\title{
A new parameter in three-term conjugate gradient algorithms for unconstrained optimization
}

\author{
Alaa Saad Ahmed ${ }^{1}$, Hisham M. Khudhur ${ }^{2}$, Mohammed S. Najmuldeen ${ }^{3}$ \\ ${ }^{1}$ Computer Science Department, College of Education for pure sciences, University of Mosul, Mosul, Iraq \\ ${ }^{2}$ Mathematics Department, College of Computer Science and Mathematics, University of Mosul, Mosul, Iraq \\ ${ }^{3}$ Ministry of Education, Kirkuk, Iraq
}

\section{Article Info}

Article history:

Received Nov 1, 2020

Revised Apr 19, 2021

Accepted Jun 18, 2021

\section{Keywords:}

Algorithm

Conjugate gradient

pure conjugacy

Unconstrained optimization

\begin{abstract}
In this study, we develop a different parameter of three term conjugate gradient kind, this scheme depends principally on pure conjugacy condition (PCC), Whereas, the conjugacy condition (PCC) is an important condition in unconstrained non-linear optimization in general and in conjugate gradient methods in particular. The proposed method becomes converged, and satisfy conditions descent property by assuming some hypothesis, The numerical results display the effectiveness of the new method for solving test unconstrained non-linear optimization problems compared to other conjugate gradient algorithms such as fletcher and revees (FR) algorithm and three term fletcher and revees (TTFR) algorithm. The numerical results demonstrate the efficacy of the suggested method for solving test unconstrained nonlinear optimization problems from where a number of iterations and evaluation of function and A comparison of the time taken to perform the functions.
\end{abstract}

This is an open access article under the CC BY-SA license.

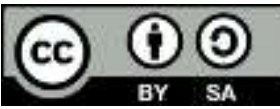

\section{Corresponding Author:}

Hisham M. Khudhur

Mathematics Department

College of Computer Science and Mathematics

University of Mosul, Iraq

Email: hisham892020@uomosul.edu.iq

\section{INTRODUCTION}

Researchers have studied the problem of unrestricted improvement as a matter of finding a solution to the minimization of the real function $f(x)$.

$$
\min _{x \in R^{n}} f(x)
$$

Whereas $f(x)$ a derivative function at least once.

Conjugate gradient (CG) algorithms are important to solve for (1) problem using the following iterative method:

$$
x_{k+1}=x_{k}+\alpha_{k} d_{k} \quad k=0,1,2
$$

Whereas $\alpha_{k}$ calculates the step size in either exactly or inexactly line search using the following relation:

$$
f\left(x_{k}+\alpha_{k} d_{k}\right)=\min _{a \geq 0} f\left(x_{k}+\alpha_{k} d_{k}\right)
$$


$d_{k+1}$ is a search direction and it is known as the following formula:

$$
\begin{array}{ll}
d_{1}=-g_{1} & k=1 \\
d_{k+1}=-g_{k+1}+\beta_{k} d_{k} & k \geq 1
\end{array}
$$

$g_{k+1}$ is a vector matrix of function $f$, and $\beta_{k}$ is a CG method parameter.

Below are parameters for some conjugate gradient algorithms:

$\beta_{k}$ is calculated with the search direction $d_{k+1}$ in the following formulas:

$$
\begin{array}{r}
d_{1}=-g_{1}, y_{k}=g_{k+1}-g_{k} \\
1-d_{k+1}=-g_{k+1}+\frac{g_{k+1}^{T} g_{k+1}}{g_{k}^{T} g_{k}} d_{k}[1] \\
2-d_{k+1}=-g_{k+1}+\frac{y_{k}^{T} g_{k+1}}{g_{k}^{T} g_{k}} d_{k}[2]
\end{array}
$$

See [3]-[8].

There are three-term conjugate gradient methods for three parameters (FR, PR, HS) proposed by Zhang [9]. These three methods always achieve regression property. Below is the search direction for some three-term conjugated gradient methods:

1) The conjugate gradient method (FR) with three-term is known as:

$$
d_{k+1}=-g_{k+1}+\beta^{F R} d_{k}-\theta_{k}^{(1)} g_{k+1}
$$

Whereas

$$
\theta_{k}^{(1)}=\frac{d_{k}^{T} g_{k+1}}{g_{k}^{T} g_{k}}
$$

2) The conjugate gradient method (PR) with three-term is known as:

$$
d_{k+1}=-g_{k+1}+\beta^{P R} d_{k}-\theta_{k}^{(2)} y_{k}
$$

Whereas

$$
\theta_{k}{ }^{(2)}=\frac{g_{k+1}^{T} d_{k}}{g_{k}^{T} g_{k}}
$$

3) The conjugate gradient method (HS) with three-term is known as:

$$
d_{k+1}=-g_{k+1}+\beta^{H S} d_{k}-\theta_{k}^{(3)} y_{k}
$$

Whereas

$$
\theta_{k}^{(3)}=\frac{g_{k+1}^{T} d_{k}}{y_{k+1}^{T} d_{k}}
$$

We notice that these methods always achieve the following property:

$$
d_{k}^{T} g_{k}=\left\|g_{k}\right\|^{2}<0 \quad \forall k
$$

Here the regression property is achieved with $c=1$. 
Often the researcher needs either exactly or inexactly line search when studying convergence and applying the CG method. Like the strong Wolf conditions.

The strong Wolf conditions are to find $\alpha_{k}$

$$
\begin{aligned}
& f\left(x_{k}+\alpha_{k} d_{k}\right) \leq f(x)+\delta \alpha_{k} g_{k}^{T} d_{k} \\
& \left|d_{k}^{T} g_{k}\left(x_{k}+\alpha_{k} d_{k}\right)\right| \leq-\sigma d_{k}^{T} g_{k}
\end{aligned}
$$

$0<\delta<\sigma<1$ are constants according to Li and Weijun [3], [9], [10].

In Section 2, we present the derivation of the new method using the FR conjugate gradient method with three-term. In Section 3, we explain the regression of the new method. In Section 4, we explain the absolute convergence of the new improved algorithm. In Section 5, the numerical results of the proposed algorithm are presented, and the performance of the new improved algorithm is compared with other algorithms in the same field.

\section{IMPROVING THE METHOD OF CONJUGATED GRADIENT FR WITH THREE-TERM}

$$
d_{k+1}=-\lambda g_{k+1}+\beta_{k}^{F R} d_{k}-\theta_{k} g_{k+1}
$$

Where $\lambda \in[0,1]$ and using pure conjugacy condition [11]

$$
\begin{aligned}
& y_{k}^{T} d_{k+1}=-\lambda y_{k}^{T} g_{k+1}+\beta_{k}^{F R} y_{k}^{T} d_{k}-\theta_{k} y_{k}^{T} g_{k+1}=0 \\
& \theta_{k} y_{k}^{T} g_{k+1}=-\lambda y_{k}^{T} g_{k+1}+\beta_{k}^{F R} y_{k}^{T} d_{k} \\
& \theta_{k}^{N E W}=-\lambda+\beta_{k}^{F R} \frac{y_{k}^{T} d_{k}}{y_{k}^{T} g_{k+1}} \\
& d_{k+1}=-g_{k+1}+\beta_{k}^{F R} d_{k}-\theta_{k}^{N E W} g_{k+1}
\end{aligned}
$$

\section{Algorithm:}

The conjugate gradient method (FR) algorithm with improved three-term:

Step 1: Let $x_{0}$ is an initial value, put $d_{0}=-g_{0}, \varepsilon>0, k=0$.

Step 2: Determine the length of the step $\alpha_{k}>0$ achieves the Wolfe conditions (5), (6).

Step 3: Determine $x_{k+1}=x_{k}+\alpha_{k} d_{k}$. If $\left\|g_{k+1}\right\|<\varepsilon$ then stopped.

Step 4: Determine $\beta_{k+1}, \theta_{k}$ from (7) and generate direction from (8).

Step 5: Put $k=k+1$. Go to step 2 .

\section{REGRESSION PROPERTY OF THE NEW FORMULA}

We will mention the proof of the sufficient descent property for the conjugate gradient method (FR) algorithm formula with improved three-term (8). The sufficient descent property for the conjugated gradient algorithm is expressed as:

$$
g_{k+1}^{T} d_{k+1} \leq-c\left\|g_{k+1}\right\|^{2} \text { for } k \geq 0 \text { and } c>0
$$

\section{Theorem (1)}

The search direction (8) with the conjugation coefficient $\beta_{k}^{F R}$ and the value of $\theta_{k}$ given by (7) will achieve (9) for all $k \geq 1$ values.

Proof: by mathematical induction

a) When $k=0$, then $d_{0}=-g_{0} \rightarrow g_{0}^{T} d_{0}=-\left\|g_{0}\right\|^{2}<0$

b) Assume that the relation $g_{k} d_{k}<0$ is true for each $k$. 
c) We prove that the relation (9) is correct when $k=k+1$ by multiplying both sides of the (8) by $g_{k+1}$. We get:

$$
\begin{aligned}
& g_{k+1}^{T} d_{k+1}=-g_{k+1}^{T} g_{k+1}+\beta_{k}^{F R} g_{k+1}^{T} d_{k}-\theta_{k}^{N E W} g_{k+1}^{T} g_{k+1} \\
& g_{k+1}^{T} d_{k+1}=-g_{k+1}^{T} g_{k+1}\left(1+\theta_{k}^{N E W}\right)+\beta_{k}^{F R} g_{k+1}^{T} d_{k}
\end{aligned}
$$

If $\theta_{k}^{N E W}>0$ then

$$
\begin{aligned}
& g_{k+1}^{T} d_{k+1}<-g_{k+1}^{T} g_{k+1}\left(1+\theta_{k}^{N E W}\right)+\beta_{k}^{F R} g_{k+1}^{T} d_{k} \\
& g_{k+1}^{T} d_{k+1}<0
\end{aligned}
$$

Thus, the regression property of the new method improved is proved.

\section{CONVERGENCE OF THE NEW IMPROVED ALGORITHM}

In this section, we will show that the three-term CG method with the coefficient of conjugation $\beta_{k}^{F R}$ and the value of $\theta_{k}$ given by (7) is absolutely convergent. We need the following assumptions to study the convergence of the new proposed algorithm:

Assumptions (A1) [10], [12]-[14]

We will impose the following assumptions on the codomain (target) function:

a) Level set $S=\left\{x \in R^{n}: f(x) \leq f\left(x_{0}\right)\right\}$ is a closed and restricted at the initial point.

b) The codomain (target) function is continuous and derivable in some proximity of $N$ of level set $s$, and its grades are continuous (lipschitz continuous). This means that there is a constant $L>0$, as that:

$$
\|g(x)-g(y)\| \leq L\|x-y\| \quad \forall x, y \in N
$$

c) The codomain (target) function $f$ is uniformly convex function, there is a constant number $g$ that achieves variance, as that:

$$
(\nabla f(x)-\nabla f(y))^{T}(x-y) \geq \mu\|x-y\|^{2} \text {, for any } x, y \in S
$$

On the other hand, using assumptions (A1) there is a positive constant $B$, as that:

$$
\begin{aligned}
& \|x\| \leq B, \forall x \in S \\
& \underline{\gamma} \leq\|g(x)\| \leq \bar{\gamma}, \forall x \in S
\end{aligned}
$$

Lemma [10], [15], [16]

We present assumptions (A1) and (10) are achieve, and by referring to (8) for the conjugate gradient where $d_{k}$ is a sloping search direction, and the length of step $\alpha_{k}$ is obtained from the strong search line for Wolfe. If

$$
\sum_{k>1} \frac{1}{\left\|d_{k+1}\right\|^{2}}=\infty
$$

we get

$$
\lim _{k \rightarrow \infty}\left(\inf \left\|g_{k}\right\|\right)=0
$$

\section{Theorem:}

We propose assumptions (A1) and (10) are accomplished by regression condition. The conjugate gradient method with the coefficient of conjugation $\beta_{k}^{F R}$ and the value of $\theta_{k}$ is given by (7), as if $\alpha_{k}$ is fulfilled with two strong wolf conditions (5) and (6). Since the codomain (target) function is uniformly convex at the plane of the set $S$, then the equation $\lim _{k \rightarrow \infty} \inf \left\|g_{k}\right\|=0$ is achieved. 
Proof:

$$
\begin{aligned}
& \left\|d_{k+1}\right\|=\left\|-g_{k+1}+\beta_{k}^{F R} d_{k}-\theta_{k}^{N E W} g_{k+1}\right\| \\
& \left\|d_{k+1}\right\| \leq\left\|g_{k+1}\right\|+\beta_{k}^{F R}\left\|d_{k}\right\|+\theta_{k}^{N E W}\left\|g_{k+1}\right\| \\
& \left\|d_{k+1}\right\| \leq\left\|g_{k+1}\right\|\left(1+\theta_{k}^{N E W}\right)+\frac{\left\|g_{k+1}\right\|^{2}}{\left\|g_{k}\right\|^{2}}\left\|d_{k}\right\| \\
& \left\|d_{k+1}\right\| \leq\left(\left(1+\theta_{k}^{N E W}\right)+\frac{\left\|g_{k+1}\right\|}{\left\|g_{k}\right\|^{2}}\left\|d_{k}\right\|\right)\left\|g_{k+1}\right\| \\
& \sum_{k \geq 1} \frac{1}{\left\|d_{k+1}\right\|} \geq\left(\frac{1}{\left(\left(1+\theta_{k}^{N E W}\right)+\frac{\left\|g_{k+1}\right\|}{\left\|g_{k}\right\|^{2}}\left\|d_{k}\right\|\right)^{2}}\right) \frac{1}{\gamma^{2}} \sum 1=\infty
\end{aligned}
$$

using the lemma above

$$
\lim _{k \rightarrow \infty}\left\|g_{k}\right\|=0
$$

\section{NUMERICAL RESULTS}

In this section, we discuss the numerical results of the new improved algorithm that we obtained from using the new formula in (8) for a set of test functions in unrestricted non-linear optimization [17]. To evaluate the performance of this proposed algorithm, the results of (75) functions [18] that were included in this study were chosen to compare with the other classical conjugate gradient method (FR, TTFR), shown in the source [17]. All codes were written using Fortran 77 and MATLAB R2009b. Using a comparison of Dolan and More' we notice through Figures 1-3 a clear superiority of the new improved algorithm with respect to the number of iterations in Figure 1 and the number of times the function is calculated in Figure 2 and also in terms of the cpu time taken to implement the program in Figure 2 in Dimensions $n=$ $100,200, \ldots, 1000$ [19]. We also wrote a Table 1 for (22) unrestricted non-linear optimization functions to

\begin{tabular}{|c|c|c|c|c|c|c|c|}
\hline \multirow[t]{2}{*}{ Problems } & \multirow[t]{2}{*}{ Dim } & \multicolumn{3}{|c|}{ Iter } & \multicolumn{3}{|c|}{ FE } \\
\hline & & NEW & FR & TTFR & NEW & FR & TTFR \\
\hline Freudenstein \& Roth & 100 & 84 & 1529 & 328 & 1979 & 44092 & 9131 \\
\hline Extended Rosenbrock & 100 & 35 & 43 & 42 & 73 & 87 & 82 \\
\hline Extended White \& Holst & 100 & 32 & 37 & 35 & 68 & 77 & 70 \\
\hline Extended Beale BEALE & 100 & 14 & 15 & 15 & 27 & 29 & 28 \\
\hline Perturbed Quadratic & 100 & 96 & 101 & 100 & 144 & 155 & 153 \\
\hline Raydan 1 & 100 & 78 & 90 & 86 & 118 & 138 & 133 \\
\hline Diagonal 2 & 100 & 63 & 64 & 71 & 105 & 105 & 121 \\
\hline Diagonal 3 & 100 & 190 & 203 & 242 & 2772 & 3075 & 4869 \\
\hline Hager & 100 & 27 & 47 & 31 & 44 & 565 & 49 \\
\hline Generalized Tridiagonal 1 & 100 & 23 & 23 & 24 & 45 & 45 & 50 \\
\hline Extended Powell & 100 & 53 & 71 & 79 & 101 & 136 & 151 \\
\hline Extended Cliff & 100 & 12 & fail & 19 & 30 & fail & 46 \\
\hline Quadratic Diagonal & 100 & 53 & 54 & 53 & 95 & 95 & 96 \\
\hline Extended Hiebert & 100 & 78 & 87 & 85 & 170 & 188 & 187 \\
\hline Extended Quadratic Penalty & 100 & 24 & 29 & 25 & 55 & 61 & 53 \\
\hline BDQRTIC & 100 & 310 & 532 & 587 & 6084 & 11664 & 11457 \\
\hline TRIDIA & 100 & 348 & 364 & 392 & 542 & 566 & 624 \\
\hline Broyden Tridiagonal & 100 & 29 & 31 & 31 & 53 & 49 & 49 \\
\hline Tridiagonal Perturbed Quadratic & 100 & 99 & 109 & 105 & 157 & 173 & 167 \\
\hline Extended DENSCHNC & 100 & 14 & 15 & 18 & 26 & 27 & 31 \\
\hline BIGGSB1 & 100 & 477 & 617 & 533 & 750 & 985 & 837 \\
\hline Extended Block-Diagonal & 100 & 14 & 15 & 15 & 24 & 26 & 26 \\
\hline
\end{tabular}
show the efficiency of the new improved method for numbers of iterations (Iter), and the number of function evaluations (FE) in Dimensions 100, with stop test $\left\|g_{k+1}\right\|<10^{-6}$ There are other research in the same field but with different test functions. For more see [5], [20]-[29].

Table 1. Unrestricted non-linear optimization functions to show the efficiency of the new improved method for numbers of iterations (Iter), and the number of function evaluations (FE) in Dimensions 100 


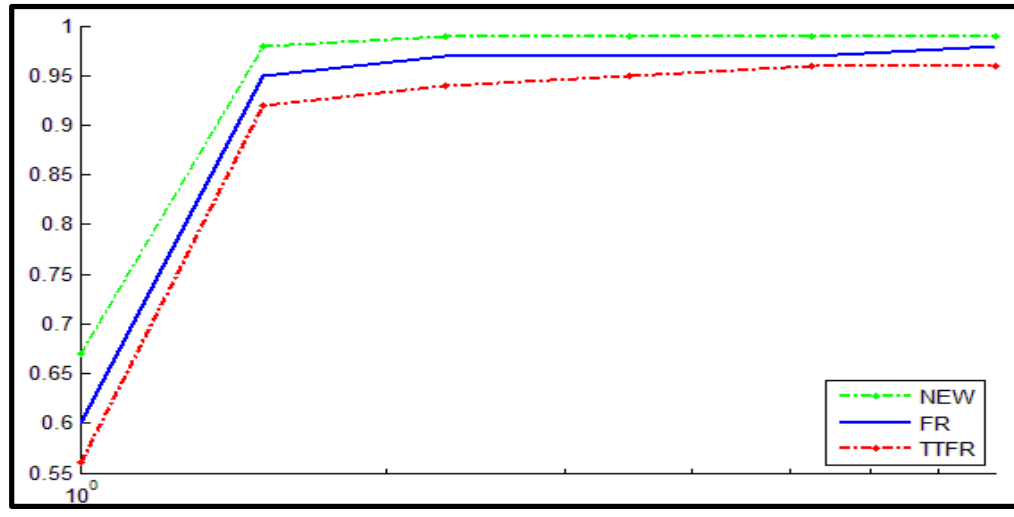

Figure 1. A comparison of the number of iterations

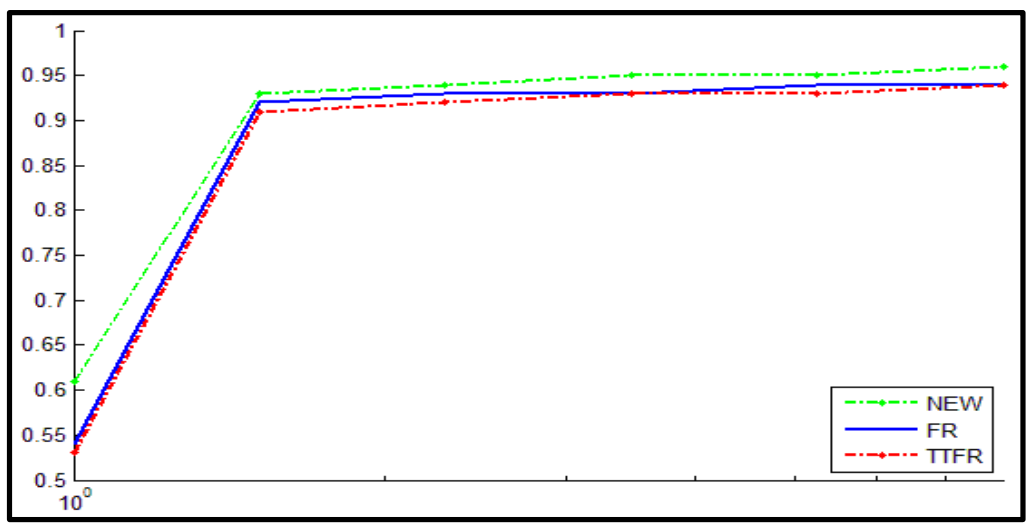

Figure 2. A comparison of the number of times a function is calculated

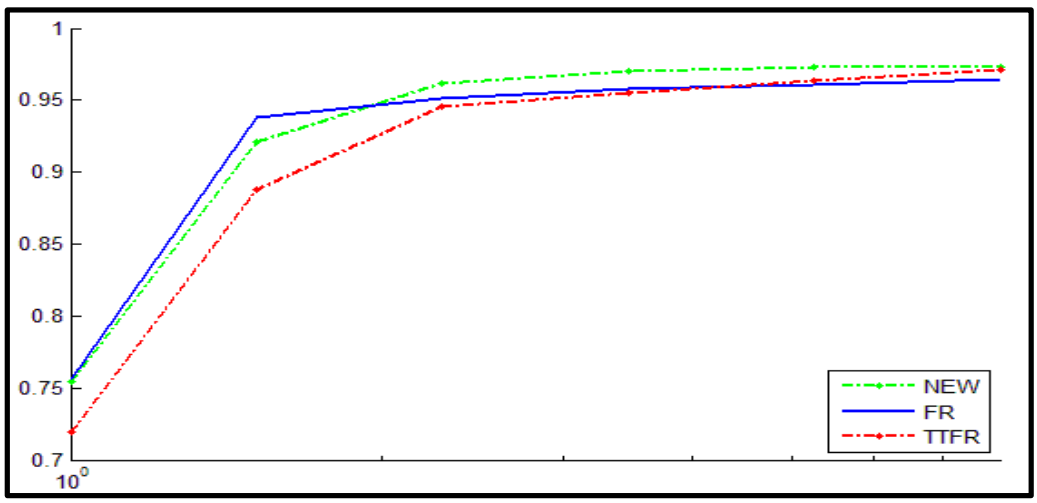

Figure 3. A comparison of the time taken to perform the functions

\section{CONCLUSIONS}

We presented in this research a new type of TTCG algorithm to solve the problems of unconstrained optimization, and the proposed algorithm has shown a high efficiency in solving these problems with the least number of iterations and with higher accuracy in reaching the approximate solution of the function.

\section{ACKNOWLEDGEMENTS}

I would like to express my sincere gratitude and appreciation to my supervisors Prof. Dr. Khalil K. Abbo for this valuable suggestion, encouragement and invaluable remark during writing this paper. 


\section{REFERENCES}

[1] R. Fletcher and C. M. Reeves, "Function minimization by conjugate gradients," Comput. J., vol. 7, no. 2, pp. 149154, 1964, doi: 10.1093/comjn1/7.2.149.

[2] Y. H. Dai and Y. Yuan, "A Nonlinear Conjugate Gradient Method with a Strong Global Convergence Property," SIAM J. Optim., vol. 10, no. 1, pp. 177-182, 1999, doi: 10.1137/S1052623497318992.

[3] H. M. Khudhur and K. K. Abbo, "A New Conjugate Gradient Method for Learning Fuzzy Neural Networks," $J$. Multidiscip. Model. Optim., vol. 3, no. 2, pp. 57-69, 2021.

[4] A. S. Ahmed, "Optimization Methods For Learning Artificial Neural Networks," University of Mosul, 2018.

[5] Hisham M. Khudhur; Khalil K. Abbo, "A New Type of Conjugate Gradient Technique for Solving Fuzzy Nonlinear Algebraic Equations," 2020.

[6] M. R. Hestenes and E. Stiefel, "Methods of conjugate gradients for solving linear systems," vol. 49, no. 1. NBS Washington, DC, 1952.

[7] L. C. W. Dixon, "Conjugate gradient algorithms: quadratic termination without linear searches," IMA J. Appl. Math., vol. 15, no. 1, pp. 9-18, 1975.

[8] E. Polak and G. Ribiere, "Note sur la convergence de méthodes de directions conjuguées," ESAIM Math. Model. Numer. Anal. Mathématique Anal. Numérique, vol. 3, no. R1, pp. 35-43, 1969.

[9] L. Zhang and W. Zhou, "Two descent hybrid conjugate gradient methods for optimization," J. Comput. Appl. Math., vol. 216, no. 1, pp. 251-264, 2008.

[10] K. K. ABBO, Y. A. Laylani, and H. M. Khudhur, "A New Spectral Conjugate Gradient Algorithm For Unconstrained Optimization,” Int. J. Math. Comput. Appl. Res., vol. 8, pp. 1-9, 2018, [Online]. Available: www.tjprc.org.

[11] H. N. Jabbar, K. K. Abbo, and H. M. Khudhur, "Four--Term Conjugate Gradient (CG) Method Based on Pure Conjugacy Condition for Unconstrained Optimization," kirkuk Univ. J. Sci. Stud., vol. 13, no. 2, pp. 101-113, 2018.

[12] H. M. Khudhur and K. K. Abbo, "New hybrid of Conjugate Gradient Technique for Solving Fuzzy Nonlinear Equations," J. Soft Comput. Artif. Intell., vol. 2, no. 1, pp. 1-8, 2021.

[13] K. K. Abbo and H. M. Khudhur, "New A hybrid Hestenes-Stiefel and Dai-Yuan conjugate gradient algorithms for unconstrained optimization," Tikrit J. Pure Sci., vol. 21, no. 1, pp. 118-123, 2016.

[14] K. K. Abbo and H. M. Khudhur, "New A hybrid conjugate gradient Fletcher-Reeves and Polak-Ribiere algorithm for unconstrained optimization," Tikrit J. Pure Sci., vol. 21, no. 1, pp. 124-129, 2016.

[15] K. K. Abbo, Y. A. Laylani, and H. M. Khudhur, "Proposed new Scaled conjugate gradient algorithm for Unconstrained Optimization," Int. J. Enhanc. Res. Sci. Technol. Eng., vol. 5, no. 7, 2016.

[16] Z. M. Abdullah, M. Hameed, M. K. Hisham, and M. A. Khaleel, "Modified new conjugate gradient method for Unconstrained Optimization," Tikrit J. Pure Sci., vol. 24, no. 5, pp. 86-90, 2019.

[17] N. Andrei, "An unconstrained optimization test functions collection," Adv. Model. Optim, vol. 10, no. 1, pp. 147$161,2008$.

[18] H. M. Khudhur, "Numerical and analytical study of some descent algorithms to solve unconstrained Optimization problems," University of Mosul, 2015.

[19] E. D. Dolan and J. J. Moré, "Benchmarking optimization software with performance profiles," Math. Program., vol. 91, no. 2, pp. 201-213, 2002.

[20] B. A. Hassan, H. O. Dahawi, and A. S. Younus, "A new kind of parameter conjugate gradient for unconstrained optimization,” Indones. J. Electr. Eng. Comput. Sci., vol. 17, no. 1, 2019, doi: 10.11591/ijeecs.v17.i1.pp404-411.

[21] B. A. Hassan and H. K. Khalo, "A new class of BFGS updating formula based on the new quasi-newton equation," Indones. J. Electr. Eng. Comput. Sci., vol. 13, no. 3, 2019, doi: 10.11591/ijeecs.v13.i3.pp945-953.

[22] B. A. Hassan, "A new type of quasi-newton updating formulas based on the new quasi-newton equation," Numer. Algebr. Control Optim., vol. 10, no. 2, 2020, doi: 10.3934/naco.2019049.

[23] B. A. Hassan, Z. M. Abdullah, and H. N. Jabbar, "A descent extension of the Dai - Yuan conjugate gradient technique,” Indones. J. Electr. Eng. Comput. Sci., vol. 16, no. 2, 2019, doi: 10.11591/ijeecs.v16.i2.pp661-668.

[24] B. A. Hassan, A. O. Owaid, and Z. T. Yasen, "A variant of hybrid conjugate gradient methods based on the convex combination for optimization," Indones. J. Electr. Eng. Comput. Sci., vol. 20, no. 2, 2020, doi: 10.11591/ijeecs.v20.i2.pp1007-1015.

[25] B. A. Hassan and M. W. Taha, "A modified quasi-Newton equation in the quasi-Newton methods for optimization," Appl. Math. Sci., vol. 13, no. 10, 2019, doi: 10.12988/ams.2019.9351.

[26] B. A. Hassan, "A new formula for conjugate parameter computation based on the quadratic model," Indones. J. Electr. Eng. Comput. Sci., vol. 13, no. 3, 2019, doi: 10.11591/ijeecs.v13.i3.pp954-961.

[27] B. A. Hassan and M. W. Taha, "A new variants of quasi-newton equation based on the quadratic function for unconstrained optimization," Indones. J. Electr. Eng. Comput. Sci., vol. 19, no. 2, 2020, doi: 10.11591/ijeecs.v19.i2.pp701-708.

[28] H. M. Khudhur, "Modified Barzilai-Borwein Method for Steepest Descent Method to Solving Fuzzy Optimization Problems (FOP)," Albahir J., vol. 12, no. 23-24, pp. 63-72, 2020.

[29] K. K Abbo and F. H Mohammed, "Spectral Fletcher-Reeves Algorithm for Solving Non-Linear Unconstrained Optimization Problems," Iraqi J. Stat. Sci., vol. 11, no. 19, pp. 21-38, 2011. 